\section{TarMed s'enlise}

\author{
R. Nyffeler
}

Dans la revue "Competence", organe officiel de $\mathrm{H}+$, son secrétaire général, le Dr Christof Haudenschild, écrit un article intitulé «TarMed est-il viable?». On peut y lire notamment: "La différence entre les représentants hospitaliers et les représentants du corps médical se réduit à la formule suivante: les hôpitaux mènent des négociations de prix tandis que les médecins mènent des négociations de revenus».

Dès le début de son article, le Dr Haudenschild pose les jalons du conflit qui va empoisonner les négociations concernant l'introduction du TarMed. Sous-jacente à son affirmation, apparait l'idée que le revenu des médecins n'a rien à voir avec les coûts réels de leurs prestations, donc le prix négocié et que notre seul but a été de majorer au maximum notre revenu, alors que les hôpitaux ne chercheront qu'à négocier le prix le plus juste pour honorer leurs prestations et que, naturellement, la notion de revenu leur est parfaitement étrangère. On nous présente là l'ange et le démon.

Plus loin, il nous dit: "La neutralité des coûts comporte le risque, qu'à l'encontre des dispositions légales, les cantons puissent ou doivent fournir des subventions aussi dans le domaine ambulatoire".

Il explique cette affirmation de la façon suivante: "Les hôpitaux ayant mené dans les négociations une politique de prix orientée sur les coûts, et le corps médical une politique de maximisation des revenus, il est désormais existentiel pour les hôpitaux que les neutralités des coûts pour les cabinets médicaux et les établissements hospitaliers soient calculées d'une façon strictement séparée. La formule doit être la suivante: la neutralité des coûts est applicable séparément pour les cabinets médicaux et pour les hôpitaux, dans le domaine LAA au niveau national et dans le domaine LAMal au niveau cantonal. Une uniformisation de la neutralité des coûts signifierait que les gains de revenus des médecins indépendants seraient financés par les hôpitaux".

Que savons-nous de TarMed? Comme on nous l'a toujours répété - et c'est pratiquement la genèse de cette œuvre colossale - le but était de rééquilibrer le

Correspondance:

Dr Richard Nyffeler

Président SMCF

Rue de l'Hôpital 15

Case postale 813

CH-1701 Fribourg chiffre d'affaires des différentes activités médicales en augmentant la rémunération des actes intellectuels au détriment de certains actes techniques. La montée au créneau du Dr Haudenschild laisse penser que H+ a déjà fait ses calculs et qu'il est clair pour eux que l'activité ambulatoire des hôpitaux comporte aujourd'hui une part d'activité technique plus importante que l'activité intellectuelle. Comme TarMed, dans sa structure même, diminue la rentabilité des actes techniques, la logique voudrait que le chiffre d'affaires des hôpitaux, pour le secteur ambulatoire, diminue, et c'est bien ce qu'il se refuse de vivre. Par un raccourci plein de naïveté, le Dr Haudenschild veut nous prouver que si le secteur ambulatoire de la médecine privée augmentait son revenu, ce serait grâce aux subventions des hôpitaux, puisque l'argent qu'il perdrait reviendrait directement, selon l'auteur, au secteur privé.

En d'autres termes, $\mathrm{H}+$ veut bien que l'on introduise une nouvelle nomenclature pour les actes médicaux, à condition que cela ne change strictement rien pour eux, sur le plan financier. C'est un peu comme si vous échangiez votre voiture économique contre une limousine grand luxe, tout en voulant consommer la même quantité de carburant pour le même nombre de kilomètres.

Si nous introduisons TarMed en sectorisant la neutralité des coûts, la valeur du point des hôpitaux sera plus haute que celle des médecins privés; il est difficile de dire aujourd'hui dans quelle proportion, mais elle pourrait être relativement importante. Ceci ne semble pas gêner $\mathrm{H}+$. A mes yeux, c'est une vision à court terme, à la fois sur le plan politique et économique.

Si cela devait arriver, certaines activités deviendraient impossibles dans le secteur privé, comme la radiologie spécialisée et l'échocardiographie par exemple, qui seraient notablement sous-évaluées. C'est peut-être le but de $\mathrm{H}+$, laisser aux braves docteurs la consultation et accaparer toute la technique. Mais on est en droit de se demander avec quels moyens financiers ils vont le faire, lorsque les instituts privés auront fermé leurs portes, le contribuable devra financer les installations nécessaires dans les hôpitaux publics. Car, aujourd'hui, du moins à Fribourg, il n'est pas possible d'effectuer l'ensemble des investigations techniques dans les seuls hôpitaux.

De plus, est-il vraiment judicieux que le secteur public soit 20 ou 30\% plus cher que le secteur privé, ceci pour une offre comparable et effectuée par des médecins ayant les mêmes formations? Si par hasard le secteur privé survivait à l'introduction du TarMed, n'y aurait-il pas le risque que les assureurs exigent, par de nouveaux contrats, que leurs clients se fassent investiguer "par le fournisseur" (puisque c'est ainsi qu'on nous appelle) le moins cher. 
En fait, les hôpitaux ont hypertrophié leur secteur ambulatoire ces dernières années, car c'était le seul rentable. Ce développement a naturellement été payé par les contribuables. On y a installé des équipements coûteux, on a ouvert parfois des cabinets médicaux, on a engagé du personnel, on a construit des bâtiments sans se soucier, naturellement, du revenu possible de ses activités, c'est du moins ce que veut nous faire croire le Dr Haudenschild, puisque lui ne négocie que des prix et se fiche royalement de ce que chaque prix mis bout à bout peut rapporter. Faut-il vraiment le croire? En fait, le problème de $\mathrm{H}+$ est que ces développements ont été possibles grâce à des subventions, que la rentabilité dépend toujours des subventions et qu'il leur sera difficile d'aller devant leur gouvernement dire que cette rentabilité risque encore de diminuer à cause de TarMed, car nous ne sommes pas naïfs au point de croire que les directeurs d'hôpitaux ne savent pas lire un bilan. Pour eux comme pour nous, le prix d'une prestation se multiplie par son volume et ceci débouche sur un chiffre d'affaires qui, lorsqu'on lui retire les charges d'exploitation et divers frais, aboutit à un bénéfice ou à une perte.

Le Dr Haudenschild nous confirme ses intentions dans son article, puisqu'il conclut en disant que «probablement, de nombreux fournisseurs de prestations dont les positions tarifaires sont moins bien évaluées par TarMed, vont tenter de maintenir leurs recettes ...".

C'est exactement ce qu'a commencé à faire $\mathrm{H}+$ et c'est bien pour ceci que TarMed s'enlise.

Dans ce contexte, la médecine privée serait bien avisée de chercher à rationaliser au maximum ses équipements et ses locaux, de favoriser les cabinets de groupe. Elle devra chercher à négocier d'autres formes de tarification que celle à l'acte. Si nous restons passifs, nous serons les perdants car, comme nous pouvons le voir, la volonté politique est clairement exprimée: on sacrifiera le secteur privé au profit du secteur public et, à moins d'une vision politique éclairée, les départements de santé publique ne s’y opposeront pas. 